\title{
Observational constraints on the cosmological evolution of dual-population radio sources
}

\author{
M. Jamrozy` \\ Radioastronomisches Institut der Universität Bonn, Auf dem Hügel 71, 53121 Bonn, Germany \\ Obserwatorium Astronomiczne, Uniwersytet Jagielloński, ul. Orla 171, 30244 Kraków, Poland \\ Received 11 March 2003 / Accepted 2 February 2004

\begin{abstract}
In this paper we show normalized differential source counts $n(S)$ at $408 \mathrm{MHz}$ and $1.4 \mathrm{GHz}$ of radio sources separately for FRI and FRII classes with extended and compact morphologies. The maps from the FIRST, NVSS, and WENSS surveys are cosmological evolution of powerful extragalactic radio sources in terms of the dual-population model (Jackson \& Wall 1999), where radio sources of Fanaroff-Riley (1974) types I and II are regarded as two physically separate types of active galactic nuclei (AGN). The predicted count values are compared with the observational data to find the best fits for the evolution and beaming parameters, and to further refine the model.
\end{abstract} \\ used to define the source morphology and flux density. The counts provide a basis for a direct test as well as constraining the
}

Key words. galaxies: active - galaxies: evolution - radio continuum: galaxies

\section{Introduction}

Galaxies (and/or quasars) hosting active galactic nuclei (AGN) are powerful extragalactic radio sources which produce jets and extended radio emitting regions (lobes) of plasma (Scheuer 1974). Most of these sources can be easily separated into two distinct classes: (i) the edge-brightened FRII sources with well-collimated jets, radio lobes and prominent hot spots, and (ii) edge-dimmed FRI sources with bending jets and peaked radio emission near the nucleus. There is a clear division in the luminosity between FRI and FRII, which lie, respectively, below and above $L_{178} \approx 2 \times 10^{25} \mathrm{~W} \mathrm{~Hz}^{-1} \mathrm{sr}^{-1}$ (Fanaroff \& Riley 1974). Owen \& Ledlow (1994) demonstrated that this separation is reproduced in the two-dimensional radiooptical luminosity plane in an unambiguous way. Furthermore, it is now quite clear that FRII and FRI sources differ radically their cosmological evolution (Wall 1980), i.e. the density and/or luminosity evolution rate of FRII is high, while that of FRI seems to be rather low.

Radio source counts $n(S)$ involving distributions of the other observational parameters (i.e. luminosity, spectral index, redshift) were used to constrain a number of numerical models of cosmological evolution of extragalactic radio sources (e.g. Wall et al. 1980; Peacock \& Gull 1981; Condon 1984; Peacock 1985; Dunlop \& Peacock 1990). All these models assumed two distinct populations of sources: steep-spectrum and flatspectrum ones, which in principle were selected based on their spectral characteristics only. Since the late 70 s, however, there have been efforts to find a common scheme for some types of

\footnotetext{
* e-mail:mjamrozy@astro.uni-bonn.de
}

extragalactic radio sources (Rowan-Robinson 1977; Scheuer \& Readhead 1979). These unification attempts aimed to explain the diversity of observational properties of extragalactic radio sources in terms of one simple model. The starting point for such models is the intrinsic structure of a radio source. Because they are not spherically symmetric, their observed form depends on the angle of view. The realization that orientation angle in AGN is of great importance for unification came with the discovery of observational superluminal motion in some compact radio sources (Cohen et al. 1971; Moffet et al. 1972) and its interpretation in terms of bulk relativistic movements of the jet's plasma (de Young 1972). It is obvious that when some emitting body moves in a relativistic manner, the radiation received by an observer is a function of the angle between the line of sight and the direction of motion. The small fraction of objects with compact morphologies are those viewed with their axes inclined at small angles to the line of sight. Furthermore, the beamed sources should be linked to an unbeamed parent population. The unified scheme of AGN has been quite successful in explaining differences between various classes of radio galaxies on the basis of the jet luminosity, its speed, relativistic beaming effect, orientation towards the observer, and environmental effects (for a review, see Urry \& Padovani 1995).

Recently, Wall and Jackson (Wall \& Jackson 1997; Jackson \& Wall 1999, hereafter WJ) introduced the dualpopulation unified scheme. Their model consists of two parent populations of powerful extragalactic radio sources, FRI and FRII, which appear (dependent on the viewing angle) as lobedominated (LD) steep-spectrum sources, or core-dominated (CD) flat-spectrum sources. 
Table 1. Observational normalized differential source counts at $1.4 \mathrm{GHz}$.

\begin{tabular}{cccccccccc}
\hline \hline & \multicolumn{2}{c}{ LD-FRI } & \multicolumn{2}{c}{ LD-FRII } & CSS & CD \\
$\Delta S$ & $\begin{array}{c}\text { Sky area } \\
{[\mathrm{sr}]}\end{array}$ & $n$ & $\begin{array}{c}\log (n(S)) \\
{\left[\mathrm{sr}^{-1} \mathrm{Jy}^{1.5}\right]}\end{array}$ & $n$ & $\begin{array}{c}\log (n(S)) \\
{\left[\mathrm{sr}^{-1} \mathrm{Jy}^{1.5}\right]}\end{array}$ & $n$ & $\begin{array}{c}\log (n(S)) \\
{\left[\mathrm{sr}^{-1} \mathrm{Jy}^{1.5}\right]}\end{array}$ & $n$ & $\begin{array}{c}\log (n(S)) \\
{\left[\mathrm{sr}^{-1} \mathrm{Jy}^{1.5}\right]}\end{array}$ \\
\hline $0.02 \div 0.05$ & 0.0133 & 25 & $1.06_{-0.10}^{+0.08}$ & 94 & $1.64_{-0.05}^{+0.04}$ & 71 & $1.51_{-0.05}^{+0.05}$ & 60 & $1.44_{-0.06}^{+0.05}$ \\
$0.05 \div 0.10$ & 0.0133 & 8 & $1.21_{-0.19}^{+0.13}$ & 47 & $1.98_{-0.07}^{+0.06}$ & 28 & $1.75_{-0.09}^{+0.08}$ & 13 & $1.42_{-0.14}^{+0.11}$ \\
$0.10 \div 0.20$ & 0.0133 & 3 & $1.22_{-0.37}^{+0.20}$ & 23 & $2.10_{-0.10}^{+0.09}$ & 19 & $2.02_{-0.11}^{+0.09}$ & 9 & $1.70_{-0.18}^{+0.12}$ \\
$0.20 \div 0.25$ & 0.0550 & 3 & $1.41_{-0.37}^{+0.20}$ & 16 & $2.14_{-0.12}^{+0.10}$ & 4 & $1.54_{-0.30}^{+0.17}$ & 8 & $1.84_{-0.19}^{+0.13}$ \\
$0.25 \div 0.55$ & 0.0906 & 10 & $1.55_{-0.15}^{+0.11}$ & 52 & $2.23_{-0.07}^{+0.05}$ & 19 & $1.79_{-0.11}^{+0.09}$ & 27 & $1.94_{-0.09}^{+0.08}$ \\
$0.55 \div 1.00$ & 0.4414 & 14 & $1.52_{-0.13}^{+0.11}$ & 93 & $2.35_{-0.05}^{+0.04}$ & 16 & $1.58_{-0.12}^{+0.10}$ & 27 & $1.81_{-0.09}^{+0.08}$ \\
$1.00 \div 2.00$ & 0.4414 & 3 & $1.21_{-0.38}^{+0.20}$ & 30 & $2.21_{-0.09}^{+0.07}$ & 11 & $1.77_{-0.15}^{+0.11}$ & 13 & $1.84_{-0.14}^{+0.11}$ \\
$2.00 \div 5.00$ & 4.30 & 20 & $1.44_{-0.11}^{+0.09}$ & 113 & $2.19_{-0.05}^{+0.04}$ & 30 & $1.62_{-0.09}^{+0.07}$ & 42 & $1.76_{-0.07}^{+0.06}$ \\
$5.00 \div 10.00$ & 4.30 & 11 & $1.83_{-0.15}^{+0.12}$ & 12 & $1.87_{-0.15}^{+0.11}$ & 6 & $1.57_{-0.23}^{+0.15}$ & 5 & $1.49_{-0.26}^{+0.16}$ \\
$10.00 \div 20.00$ & 4.30 & 1 & $1.24_{-0.74}^{+0.30}$ & 3 & $1.72_{-0.37}^{+0.20}$ & 2 & $1.54_{-0.53}^{+0.24}$ & 2 & $1.54_{-0.53}^{+0.24}$ \\
\hline
\end{tabular}

Our main goal is to test the dual-population model of FRI and FRII radio source cosmological evolution and to refine its parameters with new well-defined samples of sources at two radio frequencies and within a wide range of radio flux density. The recent radio surveys with sufficient sensitivity, spatial resolution and flux-density limit, i.e. FIRST (Becker et al. 1995), NVSS (Condon et al. 1998) and WENSS (Rengelink et al. 1997), allowed us to determine morphologies, flux densities, and spectral indices for a large enough number of objects and thereby proved to be suitable for constructing such samples.

In the following section the complete source samples used in this analysis and the counts of extragalactic radio sources $n(S)$ based on data at $1.4 \mathrm{GHz}$ and $408 \mathrm{MHz}$ are described (Sect. 2). In Sect. 3 the application of the dualpopulation numerical model of radio source evolution is shown. In Sect. 4 the results of modelling of the counts $n(S)$ compared to the observational data and discussion are given. Finally, Sect. 5 contains the summary. Throughout the paper we assume $H_{0}=50 \mathrm{~km} \mathrm{~s}^{-1} \mathrm{Mpc}^{-1}$ and the Einstein-de Sitter cosmological $\operatorname{model}\left(\Omega=\Omega_{\mathrm{m}}=1\right)$.

\section{Samples and observational source counts}

To construct observational source counts we selected two complete samples of extragalactic radio sources. The $1.4 \mathrm{GHz}$ sample is selected from four different radio surveys, namely the FIRST and Mitchell (1983) surveys, the GB/GB2 (Machalski 1998), and BDFL (Bridle et al. 1972; Bridle \& Fomalont 1974) samples. The $408 \mathrm{MHz}$ sample is based on the third Bologna radio survey (Ficarra et al. 1985). We exclude all starburst as well as galactic sources from our study. The detailed description of the $1.4 \mathrm{GHz}$ and $408 \mathrm{MHz}$ samples as well as the criterion of the source selection are described in Sects. 2.1 and 2.2, respectively.

We divide all sources from our two samples into four distinct types, i.e. (i) lobe-dominated edge-dimmed objects (LD-FRI), (ii) lobe-dominated edge-brightened objects (LD-FRII), (iii) compact objects with steep spectra (CSS), and (iv) compact objects with flat spectra (CD). A compact object is counted as CD-type when its low-frequency spectral in$\operatorname{dex} \alpha$ (if $S_{v} \propto v^{-\alpha}$, where $S_{v}$ is the flux density at frequency $v$ ) is less than 0.6 (details about spectral index determinations are given below). Our CSS-class of radio sources includes also GigaHertz-Peaked Spectrum (GPS) sources which appear in the samples. In addition, in our further analysis we treat the CSS-type objects together with the lobe-dominated FRII ones, because it is believed that they are just young counterparts of the older classical doubles (de Vries et al. 2002). Furthermore, as there is no room for lobe-dominated transient morphology (FRII/FRI) objects in the WJ scheme, we put all the "hybrid" sources into the LD-FRII class. In general, in this simple approach we have only three groups of radio sources: LD-FRI, LD-FRII and CD sources. The number of sources of a particular type within the selected $1.4 \mathrm{GHz}$ and $408 \mathrm{MHz}$ flux-density ranges is given in Tables 1 and 2, respectively.

\subsection{4 GHz sample}

The $1.4 \mathrm{GHz}$ sample consists of objects with observed flux densities between $20 \mathrm{mJy}$ and $20 \mathrm{Jy}$ (the flux scale of Baars et al. 1977 is adopted) which, depending on their flux, were selected from different surveys.

Weak source subsample

The low flux-density subsample consists of sources with $20 \leq$ $S_{1.4} \leq 200 \mathrm{mJy}$, selected from the FIRST and Mitchell (1983) radio surveys. Both surveys were made using the Very Large Array with $5^{\prime \prime}$ and $15^{\prime \prime}$ angular resolution respectively. The flux density of each object is taken from the NVSS source list. To determine morphological types of the extended radio sources we create a contour map for each of them using the FIRST and/or NVSS surveys. Next we assign by eye the particular objects in the maps to FRI or FRII type. The completeness of the FIRST and NVSS surveys is $100 \%$ in the above flux-density range, however, because of their limited baselines they are insensitive to extended structures (i.e. larger than $15^{\prime}$ in the case of NVSS). To differentiate between 
Table 2. Observational normalized differential source counts at $408 \mathrm{MHz}$.

\begin{tabular}{|c|c|c|c|c|c|c|c|c|c|}
\hline \multirow{3}{*}{$\begin{array}{c}\Delta S \\
{[\mathrm{Jy}]}\end{array}$} & \multirow{3}{*}{$\begin{array}{c}\text { Sky area } \\
{[\mathrm{sr}]}\end{array}$} & \multicolumn{2}{|c|}{ LD-FRI } & \multicolumn{2}{|c|}{ LD-FRII } & \multicolumn{2}{|r|}{ CSS } & \multicolumn{2}{|r|}{$\mathrm{CD}$} \\
\hline & & $n$ & $\log (n(S))$ & $n$ & $\log (n(S))$ & $n$ & $\log (n(S))$ & $n$ & $\log (n(S))$ \\
\hline & & & {$\left[\mathrm{sr}^{-1} \mathrm{Jy}^{1.5}\right]$} & & {$\left[\mathrm{sr}^{-1} \mathrm{Jy}^{1.5}\right]$} & & {$\left[\mathrm{sr}^{-1} \mathrm{Jy}^{1.5}\right]$} & & {$\left[\mathrm{sr}^{-1} \mathrm{Jy}^{1.5}\right]$} \\
\hline $0.10 \div 0.20$ & 0.0102 & 12 & $1.94_{-0.15}^{+0.11}$ & 62 & $2.65_{-0.06}^{+0.05}$ & 29 & $2.32_{-0.09}^{+0.07}$ & 13 & $1.97_{-0.14}^{+0.11}$ \\
\hline $0.20 \div 0.40$ & 0.0327 & 10 & $1.81_{-0.16}^{+0.12}$ & 91 & $2.76_{-0.05}^{+0.04}$ & 44 & $2.45_{-0.06}^{+0.06}$ & 14 & $1.95_{-0.14}^{+0.10}$ \\
\hline $0.40 \div 0.80$ & 0.0785 & 9 & $1.84_{-0.18}^{+0.12}$ & 95 & $2.86_{-0.05}^{+0.04}$ & 50 & $2.58_{-0.07}^{+0.06}$ & 16 & $2.09_{-0.13}^{+0.09}$ \\
\hline $0.80 \div 1.60$ & 0.1773 & 9 & $1.94_{-0.18}^{+0.12}$ & 81 & $2.89_{-0.06}^{+0.05}$ & 31 & $2.47_{-0.09}^{+0.07}$ & 17 & $2.21_{-0.12}^{+0.10}$ \\
\hline $1.60 \div 5.00$ & 0.2774 & 5 & $1.85_{-0.25}^{+0.16}$ & 58 & $2.92_{-0.06}^{+0.05}$ & 15 & $2.33_{-0.13}^{+0.10}$ & 10 & $2.15_{-0.18}^{+0.12}$ \\
\hline $5.00 \div 25.00$ & 0.2774 & 2 & $2.18_{-0.53}^{+0.23}$ & 10 & $2.88_{-0.17}^{+0.12}$ & 3 & $2.35_{-0.37}^{+0.20}$ & 0 & $<1.88$ \\
\hline
\end{tabular}

compact steep-spectrum (CSS) and flat-spectrum (CD) sources we calculate a spectral index between $325 \mathrm{MHz}$ (WENSS) and $1.4 \mathrm{GHz}$ (NVSS). In some cases when the source is not included in the WENSS catalog, its low-frequency flux density is taken from other radio catalogs. Because the spectral information about sources comes from the flux density measurements performed at two frequencies only, some of the compact GigaHertz-Peaked Spectrum sources could be erroneously classified as CD ones. However, the fraction of GPS sources at $1.4 \mathrm{GHz}$ constitutes at most only about $5 \%$ of the entire sample (see Table 1 of Jackson \& Wall 1999).

The sources from the FIRST survey are selected from a small region of the sky between $07^{\mathrm{h}} 19^{\mathrm{m}}<\alpha_{2000}<08^{\mathrm{h}} 00^{\mathrm{m}}$ and $+28^{\circ} 45^{\prime}<\delta_{2000}<+31^{\circ} 10^{\prime}$. The sources from the Mitchell survey are selected from a sky region between $12^{\mathrm{h}} 56^{\mathrm{m}}<\alpha_{2000}<$ $13^{\mathrm{h}} 20^{\mathrm{m}}$ and $+28^{\circ} 16^{\prime}<\delta_{2000}<+32^{\circ} 02^{\prime}$. There are 400 radio sources in this subsample.

\section{Intermediate source subsample}

This subsample is taken from the GB/GB2 revised list whose definition is given by Machalski (1998) and consists of 346 sources with fluxes between $200 \mathrm{mJy}$ and $2 \mathrm{Jy}$. All sources have $b_{\mathrm{II}}>20^{\circ}$. The value of each source's spectral index and the shape of the spectrum is taken from Machalski (1998).

\section{Strong source subsample}

The subsample of 247 sources with flux densities $2 \leq$ $S_{1.4}<20 \mathrm{Jy}$, found within the area of the sky defined by $-5^{\circ}<\delta_{2000}<+70^{\circ}, b_{\mathrm{II}}>20^{\circ}$, is taken from BDFL sample. The original source's flux has been transformed from the KPW (Kellermann et al. 1969) to the Baars scale $\left(S_{\mathrm{KPW}} \times 1.029=S_{\text {Baars }}\right)$. In this subsample the spectral index is taken from the Kühr catalogue (Kühr et al. 1981).

\section{2. $408 \mathrm{MHz}$ sample}

The $408 \mathrm{MHz}$ sample consists of 686 extragalactic sources with flux $0.10 \leq S_{408}<25$ Jy selected from the B3-VLA catalogue (Vigotti et al. 1989). The original $408 \mathrm{MHz}$ flux densities are taken from The Third Bologna Catalogue (Ficarra et al. 1985). The sample includes objects within the RA range of $07^{\mathrm{h}} 03^{\mathrm{m}}<\alpha_{1950}<15^{\mathrm{h}} 00^{\mathrm{m}}$. The spectral index for sources from this sample is determined between $408 \mathrm{MHz}$ (B3) and $1.4 \mathrm{GHz}$ (NVSS).

\subsection{Source counts}

The observational normalized differential source counts $n(S)$ are defined as:

$n(S)=\frac{n A^{-1}}{\Delta S S^{-2.5}}$

where $n$ is the number of sources (e.g. of lobe dominated FRI or FRII, or of core dominated ones) in the sky area $A$ with flux density between $S_{1}$ and $S_{2}\left(\Delta S=S_{2}-S_{1}\right)$, and

$S=\exp \left\{\left(\log \left(S_{1}\right)+\frac{\log \left(S_{2}\right)-\log \left(S_{1}\right)}{2}\right) \times \ln (10)\right\}$.

The errors $\Delta n(S)$ are derived as:

$\Delta n(S)=\left|\frac{(n \pm \sqrt{n}) A^{-1}}{\Delta S S^{-2.5}}-n(S)\right|$.

We also determine the observational fraction $F$ of sources of a particular morphology within a given flux-density range $(\Delta S)$,

$F=\frac{n}{n_{\Delta S}}$

and $(0 \leq F \leq 1)$, where $n_{\Delta S}$ is the number of all sources in the same flux-density range. The error of the fraction $F$ is derived as:

$\Delta F=\frac{F(1-F)}{\sqrt{n}}$.

The sources from the samples described in Sect. 2 are used to construct the separate radio source distributions of coredominated and lobe-dominated FRI or FRII objects. The resulting normalized differential counts $n(S)$ at $1.4 \mathrm{GHz}$ and $408 \mathrm{MHz}$ are presented in Tables 1 and 2, respectively. The columns give the adopted flux-density intervals, the area of sky, the observed number of sources of particular morphology and the relevant differential source counts with estimated errors. We are aware that our procedure of morphological classification of radio sources is not quite perfect and may occasionally lead to erroneous assignments. The misclassified cases can have a significant effect on the resulting counts when a number of objects within the given interval offlux density is small. Figures 1 and 2 

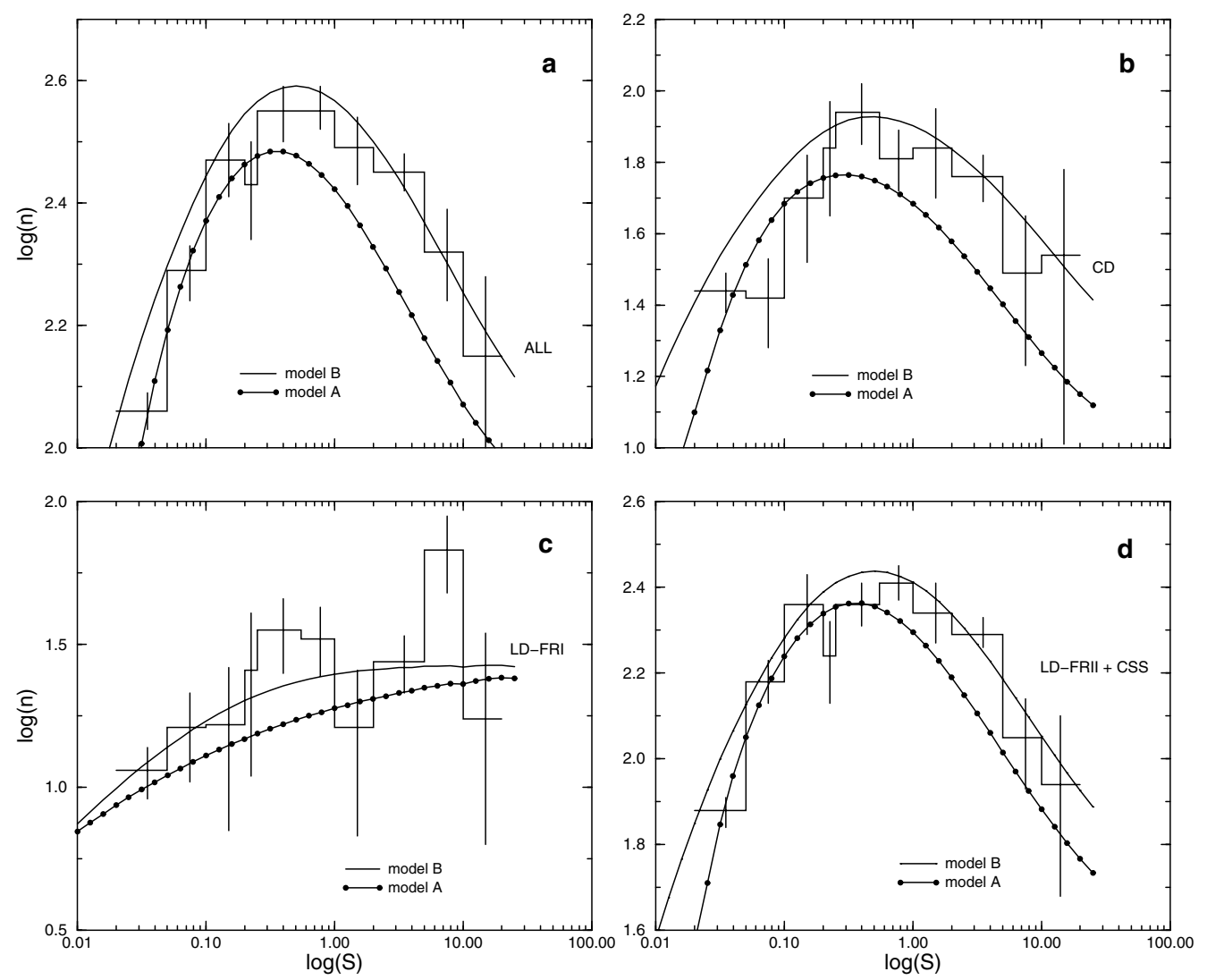

Fig. 1. Normalized differential counts of sources of different morphological types at $1.4 \mathrm{GHz}$. Abscissa: $\log$ flux-density (Jy). Ordinate: log differential number of sources multiplied by $S^{2.5}\left[\mathrm{sr}^{-1} \mathrm{Jy}^{1.5}\right]$. Observational data are provided in the form of stepped curve with vertical error bars. The solid curve shows the counts predicted from model B and the dotted solid one - counts from model A. The respective panels give the counts for a) all sources in the sample; b) compact FRI and compact FRII sources; c) FRI lobe-dominated sources; and d) FRII lobe-dominated and CSS sources, (for details, see the text).

show the counts described above, while in Fig. 3, the observed source fraction $F$, both at $1.4 \mathrm{GHz}$ and $408 \mathrm{MHz}$, are plotted.

\section{Dual-population model}

The dual-population paradigm for powerful extragalactic radio sources, proposed by WJ, involves two parent populations: FRII and FRI type sources. The orientation angle of the radio axis to the plane of the sky determines the observed characteristics and classification of the objects as lobe-dominated steep-spectrum sources or core-dominated flat-spectrum ones. The beaming model is applied to those distinct populations to model their respective contributions to the source counts. The evolution and beaming scheme can be used to predict the intensity-dependent $n(S)$ distributions for beamed and unbeamed counterparts of FRI and FRII radio source populations at any frequency. The consecutive steps to model the $n(S)$ counts are: 1) constructing the local luminosity function and assuming a form for the evolution function; 2) using the beaming model to compute the flux-densities and spectral indices of modeled sources; 3 ) computing the expected source counts and comparing them to the observational data; and 4) varying the values of model parameters and repeating the steps above until the predicted counts fit well the observations. The above procedure is applied to FRI and FRII sources separately.

\subsection{Radio luminosity function and evolution function}

The radio luminosity function $\rho(L, z, v) \mathrm{d} L$ specifies the comoving number density of sources with luminosities $L$ to $L+\mathrm{d} L$ measured at frequency $v$ (in the source frame) and which are at redshift $z$. The local luminosity function $\rho\left(L, z_{0}, v\right)$ along with an evolution function $E(L, z)$, which modifies the former one, give the radio luminosity function at any epoch $\rho(L, z, v)=$ $\rho\left(L, z_{0}, v\right) E(L, z)$. We adopt the form of the local radio luminosity function directly from WJ. The evolution function $E(L, z)$ is chosen as an exponential luminosity-dependent density evolution, first proposed by Wall et al. (1980). The evolution function has the form:

$$
E(L, z)=\exp M(L) \tau(z)
$$

where $\tau$ is the so-called "look-back" time given by

$\tau(z)=1-(1+z)^{-1.5}$.

We also apply a redshift cutoff $z_{\mathfrak{c}}$, so that for $z>z_{\mathfrak{c}}, E(L, z)=0$. The evolution rate $M$ depends on a source radio power $L$ :

$\begin{array}{ll}M(L)=M_{\max } \frac{\log L-\log L_{1}}{\log L_{2}-\log L_{1}} & \text { for } L_{1} \leq L \leq L_{2}, \\ M(L)=0 & \text { for } L<L_{1}, \\ M(L)=M_{\max } & \text { for } L>L_{2} .\end{array}$



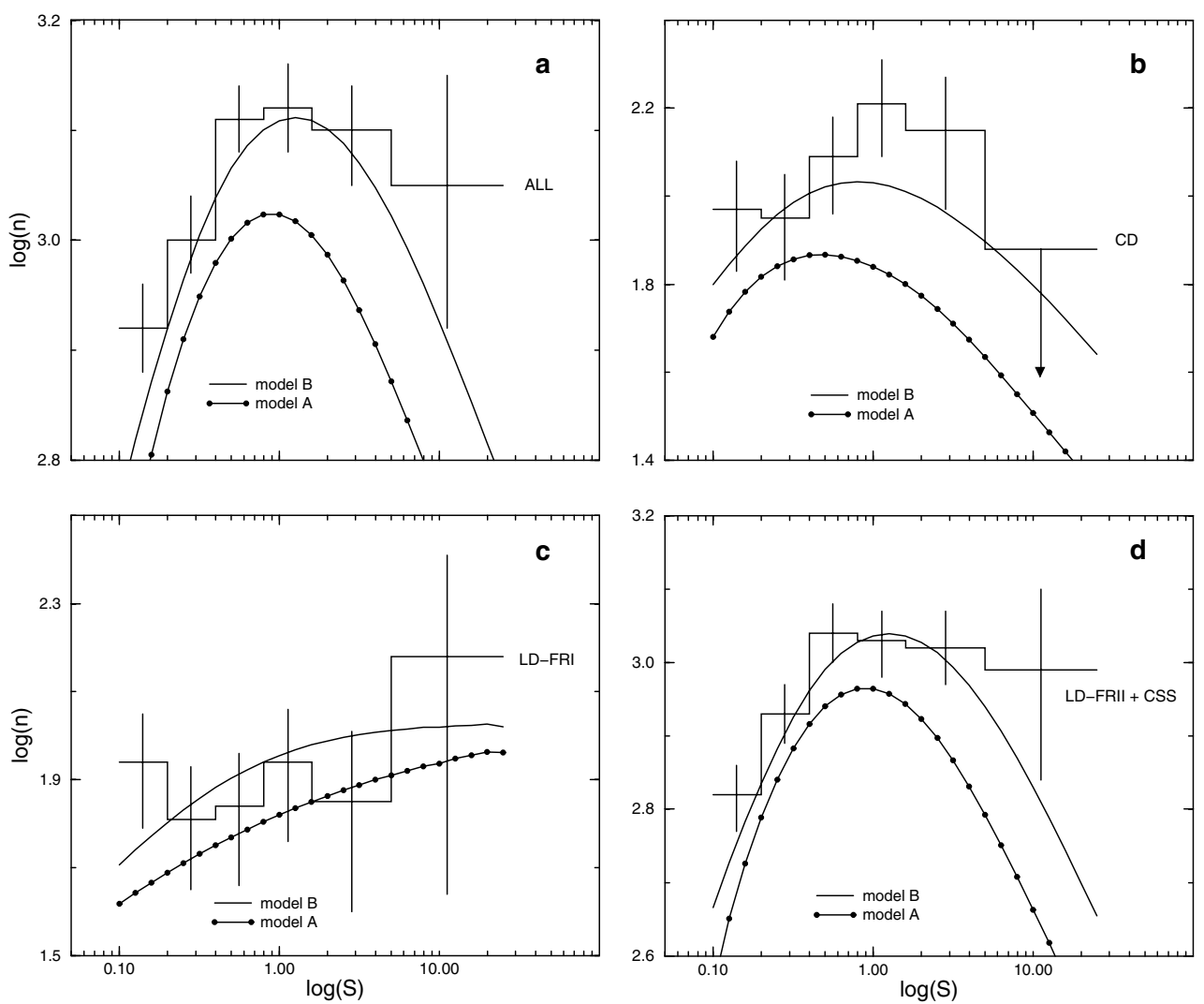

Fig. 2. Normalized differential counts of sources of different morphological types at $408 \mathrm{MHz}$. Abscissa: log flux-density (Jy). Ordinate: $\log$ differential number of sources multiplied by $S^{2.5}\left[\mathrm{sr}^{-1} \mathrm{Jy}^{1.5}\right]$. Observational data are provided in the form of stepped curve with vertical error bars. The solid curve shows the counts predicted from model B and the dotted solid one - counts from model A. The respective panels give the counts for a) all sources in the sample; b) compact FRI and compact FRII sources; c) FRI lobe-dominated sources; and d) FRII lobe-dominated and CSS sources, (for details, see the text).

\subsection{Relativistic beaming}

In the beaming scenario, it is assumed that all radio sources are intrinsically steep-spectrum, and flat-spectrum sources are merely those in which the core radiation is boosted in a sufficiently strong way to dominate the total flux density. The beaming model predicts that the observed radio flux density $S_{v}$ from a given source at a given frequency $v$ is composed of flatspectrum emission from the core and steep-spectrum from the extended lobes emission, $S_{\nu \mathrm{CD}}$ and $S_{\nu \mathrm{LD}}$ respectively. The observed core-to-extended flux ratio $R$, which depends on the the transverse (unbeamed) core dominance parameter, line-of-sight orientation of the jet towards the observer and the jet's speed, is given by:

$$
\begin{aligned}
R(\theta, v) & \equiv \frac{S_{\nu \mathrm{CD}}}{S_{\nu \mathrm{LD}}} \\
& =R_{\mathrm{c}}(v)\left\{[\gamma(1-\beta \cos \theta)]^{-\mathrm{p}}+[\gamma(1+\beta \cos \theta)]^{-\mathrm{p}}\right\},
\end{aligned}
$$

where $R_{\mathrm{c}}(v)$ is the frequency-dependent intrinsic core-toextended flux ratio, $\theta\left(0^{\circ} \leq \theta \leq 90^{\circ}\right)$ is the angle between the jet axis and line-of-sight. $\gamma$ is the Lorentz factor of the plasma, $\gamma \equiv \frac{1}{\sqrt{1-\beta^{2}}}$. For the radio emission with continuously ejected plasma, $p=2-\alpha_{\mathrm{CD}}$, where $\alpha_{\mathrm{CD}}$ is the spectral index of core emission, for which we assume the value of 0.0. Following WJ, we consider that the mean spectral index is $\alpha_{\mathrm{LD}}=0.75$, and the total flux density of a source is
$S_{v}=S_{\nu \mathrm{CD}}+S_{v \mathrm{LD}}=S_{\nu \mathrm{LD}}(R(\theta, v)+1)$. The flux densities of the flat-spectrum component at two frequencies $v_{1}$ and $v_{2}$ are equal $\left(S_{v_{1} \mathrm{CD}}=S_{v_{2} \mathrm{CD}}\right)$. The parameters of $\gamma$ and $R_{\mathrm{c}}(v)$ are the same for each object from a given population of radio sources. A source is classified either as a core-dominated one (CD) if $\theta$ is small enough so that spectral index of the entire source is $\alpha_{v_{1}}^{\nu_{2}} \leq 0.6$, or a lobe-dominated one (LD) for $\alpha_{v_{1}}^{\nu_{2}}>0.6$. This spectral index is given by the equation (Morisawa \& Takahara 1987):

$\alpha_{v_{1}}^{v_{2}}=\frac{\log \left[\left(\frac{v_{1}}{v_{2}}\right)^{\alpha_{\mathrm{LD}}} \frac{R\left(\theta, v_{2}\right)+1}{R\left(\theta, v_{1}\right)+1}\right]}{\log \frac{v_{1}}{v_{2}}}$.

The critical observed core-to-extended flux ratio $R_{\min }(\theta, v)$, dividing the sources into the CD or LD type at a frequency $v_{2}$ is given by:

$R_{\min }\left(\theta, v_{2}\right)=\frac{\left(\frac{v_{1}}{v_{2}}\right)^{\alpha_{v_{1}}^{v_{2}}-\alpha_{\mathrm{LD}}}-1}{1-\left(\frac{v_{1}}{v_{2}}\right)^{\alpha_{v_{1}}^{\nu_{2}}}}$

$R_{\min }(\theta, v)$ corresponds to the critical angle $\theta$, which can be determined from equation:

$\cos (\theta)=\frac{1}{\beta}-\frac{1}{\beta \gamma}\left(\frac{R_{\mathrm{c}}(v)}{R_{\min }}\right)^{p^{-1}}$,

assuming that the beaming due to the counter-jet is negligible. 

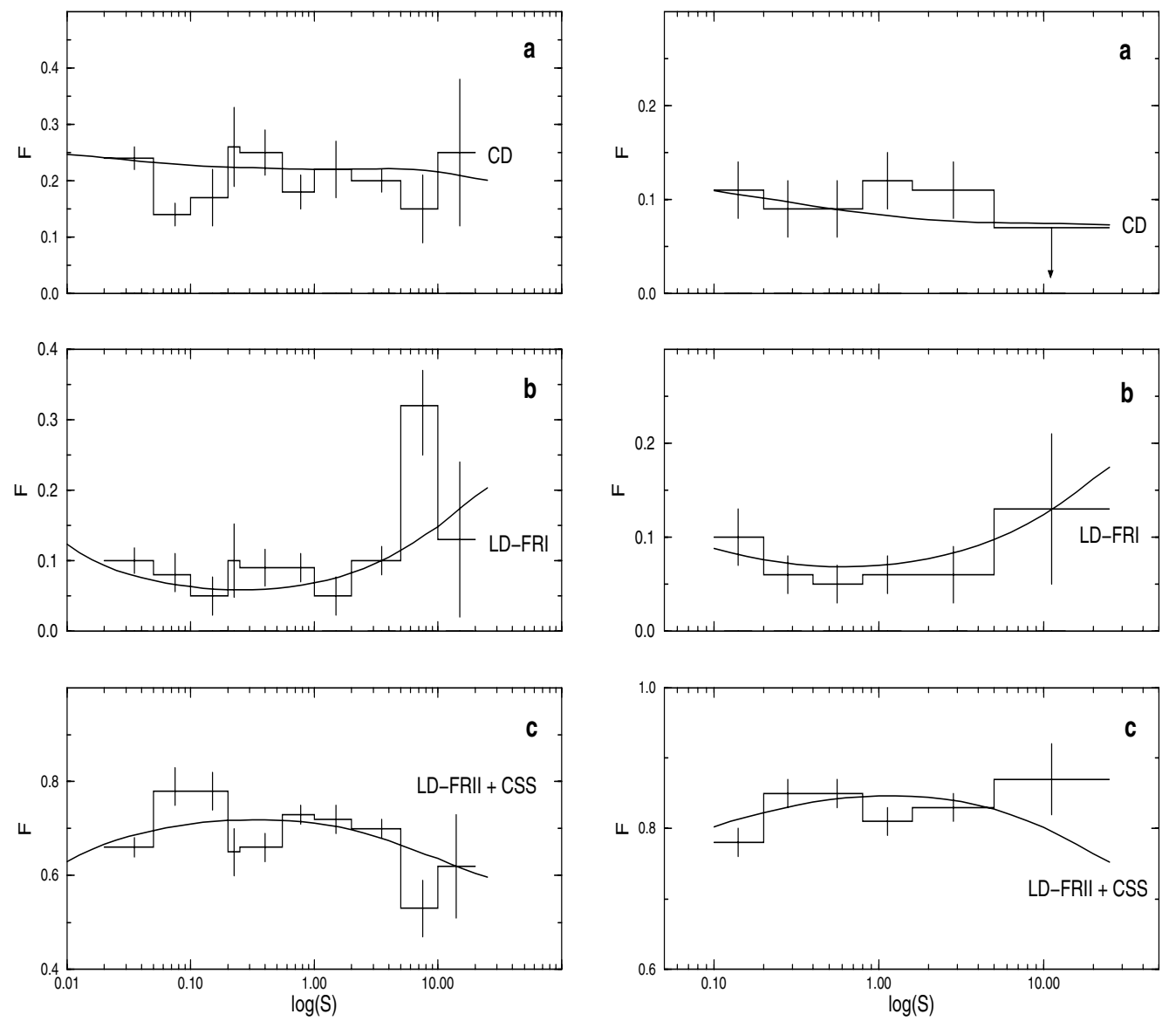

Fig. 3. Fraction of a) compact flat-spectrum sources; b) FRI lobe-dominated sources; and c) FRII lobe-dominated together with CSS sources in the sample at $1.4 \mathrm{GHz}$ - left panels, and at $408 \mathrm{MHz}$ - right panels. The solid curve shows the source ratio predicted from model $\mathrm{B}$.

\subsection{Expected $n(S)$ distributions}

The number of sources with flux-densities $S$ to $S+\mathrm{d} S$ at frequency $v$ are given by:

$n(S, v)=\int_{z=0}^{z_{\max }} \int_{R_{\min }}^{R_{\max }} n(S, z, v) P(R(\theta, v)) \mathrm{d} R \mathrm{~d} z$,

where $n(S, z, v) \mathrm{d} S$ is the number of sources in $4 \pi$ sr of the sky with flux-densities $S$ to $S+\mathrm{d} S$ at frequency $v$ and redshift $z$. $P(R)$ is the distribution of $R(\theta, v)$ and in a sample of randomly orientated radio sources with the same values of $R_{\mathrm{c}}(v)$ and $\gamma$, $P(R(\theta, v))$ is given by:

$P(R(\theta, v)) \mathrm{d} R=\mathrm{d}(\cos \theta)$,

so Eq. (9) becomes:

$n(S, v)=\int_{z=0}^{z_{\max }} \int_{\theta_{\min }}^{\theta_{\max }} n(S, z, v) \sin \theta \mathrm{d} \theta \mathrm{d} z$.

The values of $\theta_{\min }$ and $\theta_{\max }$ depend on the source morphology. For CD sources $\theta_{\min }=0^{\circ}$, and $\theta_{\max }=90^{\circ}$ for LD sources. $\theta_{\max }$ for CD is equal to $\theta_{\min }$ for LD sources and can be derived from Eq. (8).

The radio luminosity function $\rho(L, z, v)$ and the number of sources $n(S, z, v)$ are related in the following way:

$n(S, z, v) \mathrm{d} S=\rho(L(S), z, v) \mathrm{d} L \mathrm{~d} V$ where $\mathrm{d} V$ is a volume element and is equal to $4 \pi D^{2} \mathrm{~d} r$.

$D=\frac{2 c\left\{\Omega z+(\Omega-z)\left[(\Omega z+1)^{\frac{1}{2}}-1\right]\right\}}{H_{0}(1+z) \Omega^{2}}$

and

$\mathrm{d} r=\frac{c \mathrm{~d} z}{H_{0}(1+z)(1+\Omega z)^{\frac{1}{2}}}$.

Inserting $L=D^{2}(1+z)^{1+\alpha} S$ and Eq. (13) into Eq. (11) yields:

$n(S, z, v)=\frac{4 \pi D^{4} c(1+z)^{\alpha} \rho(L, z, v)}{H_{0}(1+\Omega z)^{\frac{1}{2}} L}$.

It is useful to calculate the normalized counts and for that purpose we multiply Eq. (14) by $S^{\frac{5}{2}}$ :

$n(S, z, v) S^{\frac{5}{2}}=\frac{4 \pi c(1+z)^{-\frac{5}{2}-\frac{3}{2} \alpha} \rho(L, z, v) L^{\frac{3}{2}}}{H_{0}(1+\Omega z)^{\frac{1}{2}} D}$.

\section{Results and discussion}

Following the procedure given in the preceding section we derive the normalized differential counts and the source fractions. The set of parameter values originally proposed by WJ is called "model A" (see Table 3). It is necessary to modify these values to fit the observational data. The best-modeled fit to the source 
Table 3. Values of beaming and evolution parameters for models A and $\mathrm{B}$.

\begin{tabular}{lrrrr}
\hline \hline Parameter & \multicolumn{2}{c}{ FRI } & \multicolumn{2}{c}{ FRII } \\
& \multicolumn{1}{c}{$\mathrm{A}$} & \multicolumn{1}{c}{$\mathrm{B}$} & \multicolumn{1}{c}{$\mathrm{A}$} & \multicolumn{1}{c}{$\mathrm{B}$} \\
\hline$\gamma$ & $10.3(\dagger)$ & 10.3 & $20.0(\dagger)$ & 20.0 \\
$R_{\mathrm{c}}(v)(*)$ & $0.010(\dagger)$ & 0.010 & $0.004(\dagger)$ & 0.006 \\
$\log \left(L_{1}(* *)\right)$ & - & 26.50 & $25.44(\ddagger)$ & 24.50 \\
$\log \left(L_{2}(* *)\right)$ & - & - & $27.34(\ddagger)$ & 27.95 \\
$M_{\max }$ & - & 5.0 & $10.93(\ddagger)$ & 12.20 \\
$z_{\max }$ & $5.0(\ddagger)$ & 5.0 & $5.62(\ddagger)$ & 5.62 \\
\hline
\end{tabular}

$\dagger$ The value is taken from Wall \& Jackson (1997), and

$\$$ the value is taken from Jackson \& Wall (1999).

(*) $v=5 \mathrm{GHz},(* *) \mathrm{W} \mathrm{Hz}^{-1} \mathrm{sr}^{-1}$.

counts at $1.4 \mathrm{GHz}$ and $0.408 \mathrm{GHz}$ is called "model B" and its parameter values are given also in Table 3 . The values are determined using statistical methods, by minimizing $\chi^{2}$ evaluated between the observed and modeled source distributions, both at 1.4 and $0.408 \mathrm{GHz}$. The value of $\chi^{2}$ is evaluated as:

$\chi^{2}=\sum_{i=1}^{N}\left(\frac{n_{\text {data }_{i}}-n_{\text {model }_{i}}}{\operatorname{err}_{\text {data }_{i}}}\right)^{2}$,

where $n_{\mathrm{data}_{i}}$ is the value of the data in the $i$ th bin, similarly $n_{\text {model }_{i}}$ and $\operatorname{err}_{\text {data }_{i}}$ are the model value and data error in the $i$ th bin, respectively. These all are determined in the logarithm space and the sum is taken over the $N$ bins.

The observed and calculated $n(S)$ distributions for 1.4 and $0.408 \mathrm{GHz}$ are presented in Figs. 1 and 2, respectively. Figure 3 shows the expected and observed source fraction.

The best-fitted parameters of cosmological evolution and relativistic beaming that we estimate are slightly different from those obtained by $\mathrm{WJ}$ on the basis of the $5 \mathrm{GHz}$ data. However, it is important to note that our calculations do not strictly follow all the steps proposed by Jackson \& Wall (1999). For example, we do not demand the evolution function $E(L, z)$ to peak at $z_{\mathrm{c}} / 2$. Furthermore, our approach does not involve any dependence of the intrinsic core-to-extended flux ratio $R_{\mathrm{c}}$ on radio power. We estimate that the transition between evolving and non-evolving FRII sources appears for luminosities $L_{151}=10^{24.5}\left(\mathrm{~W} \mathrm{~Hz}^{-1} \mathrm{sr}^{-1}\right)$, whereas objects with $L_{151}>$ $10^{27.95}\left(\mathrm{~W} \mathrm{~Hz}^{-1} \mathrm{sr}^{-1}\right)$ show maximal evolution with the rate of $M_{\max }=12.2$.

To fit the FRI observational data, it is necessary to introduce some luminosity evolution for these type of sources. However, Urry \& Padovani (1995) report that the behavior of FRI radio galaxies is consistent with no evolution. Also, in the WJ scheme, the cosmological evolution of FRI does not explicitly appear, however, there are no fundamental aspects in their model forbidding the evolution of FRI sources in general. The idea that FRI should evolve is confirmed by the recent work of Snellen \& Best (2001), who on inspecting Hubble Deep Field images came across two sources of FRI morphology. This could mean that this kind of source is significantly more abundant at high redshifts than at the present epoch. They suggest also that cosmological evolution of FRI sources could be similar to that of FRII (i.e. is radio luminosity dependent). We propose that the evolution of FRI sources is similar to that of FRII, (Sect. 3.1, Eq. (4)). The evolution rate $M$, which depends on luminosity, has the form:

$$
\begin{array}{ll}
M(L)=0 & \text { for } L<L_{1}, \\
M(L)=M_{\max } & \text { for } L \geq L_{1} .
\end{array}
$$

We estimate that the small fraction of FRI with luminosities $L_{151} \geq 10^{26.5}\left(\mathrm{~W} \mathrm{~Hz}^{-1} \mathrm{sr}^{-1}\right.$ ) evolve with cosmic time. This is supported by the fact that at $L_{151} \approx 10^{26.5}\left(\mathrm{~W} \mathrm{~Hz}^{-1} \mathrm{sr}^{-1}\right)$ in the low-frequency samples the fraction of objects with observed broad-line nuclei changes radically from $\sim 0.4$ at higher radio luminosities to $\sim 0.1$ at lower luminosities (Willott et al. 2000).

The best-fit relativistic beaming parameters $\left(R_{\mathrm{c}}(v)\right.$ and $\left.\gamma\right)$ obtained are similar to those estimated by Wall \& Jackson (1997). They are also in agreement with those derived from observations. The observed core-to-extended flux ratio of individual sources spans a wide range of values from $\sim 10^{-5}$ for the lobe-dominated sources (Morganti et al. 1993) to $\sim 10^{3}$ for the core-dominated sources (Murphy et al. 1993). The observations show also that there is a large spread of Lorentz factor $\gamma$ values from 2 to 20 (Vermulen \& Cohen 1994), while some numerical simulations give $4 \leq \gamma \leq 40$ (Urry \& Padovani 1995). However, the Lorentz factor value of FRII sources should be greater from those of FRI (Urry \& Padovani 1995).

Some recent papers (e.g. Caccianiga et al. 2002) suggest the existence of another class of sources with compact morphologies that do not originate from FRI or FRII populations. Our investigation shows that there is no explicit need to introduce any additional population of compact powerful radio sources, apart from the beaming version of FRI and FRII parent populations. However, if the values of beaming parameters given here are overestimated, then the model predictions for compact sources will be too small when compared to the data.

\section{Summary}

In this paper we have presented the observational $\log (n)-$ $\log (S)$ relation constructed for powerful extragalactic radio sources separately for different morphological classes (i.e. FRII and FRI lobe-, and core-dominated). The counts are performed over three flux-density decades (from $20 \mathrm{mJy}$ to $20 \mathrm{Jy}$ ). We compare the observational source distributions with the predictions of the dual-population WJ model. From our analysis it can be inferred that:

(i) the WJ dual-population evolutionary scheme enables predictions that are in good agreement with the observational counts of powerful extragalactic radio sources of a given morphological type;

(ii) we found a satisfactory set of physical parameter values for relativistic beaming and cosmological evolution which in the latter case differ slightly from those estimated by $\mathrm{WJ}$;

(iii) to fit properly the observational data of FRI sources it is necessary to introduce an extra term of positive cosmological evolution, i.e. in the proposed simple approach the most luminous FRI sources evolve in a similar way to the FRII sources. 
Acknowledgements. We are grateful to Prof. Machalski and Dr. Ryś for their helpful comments and suggestions. We also warmly thank the referee for helpful suggestions which have been very useful in improving and clarifying this paper. We acknowledge the Deutsche Forschungsgemeinschaft for the award of a postdoctoral felowship (GRK787).

\section{References}

Baars, J. W. M., Genzel, R., Pauliny-Toth, I. I. K., et al. 1977, A\&A, 61,99

Becker, R. H., White, R. L., \& Helfand, D. J. 1995, ApJ, 450, 559

Bridle, A. H., \& Fomalont, E. B. 1974, AJ, 79, 1000

Bridle, A. H., Davis, M. N., \& Fomalont, E. B. 1972, AJ, 77, 405

Caccianiga, A., Marcha, M. J., Anton, S., et al. 2002, MNRAS, 329, 877

Cohen, M. H., Cannon, W., Purcell, G. H., et al. 1971, ApJ, 170, 207

Condon, J. J., Cotton, W. D., Greisen, E. W., et al. 1998, AJ, 115, 1693

Condon, J. J. 1984, ApJ, 287, 461

Dunlop, J. S., \& Peacock, J. A. 1990, MNRAS, 247, 19

Fanaroff, B. L., \& Riley, J. M. 1974, MNRAS, 167, 31

Ficarra, A., Grueff, G., \& Tomassetti, G. 1985, A\&AS, 59, 255

Jackson, C. A., \& Wall, J. V. 1999, MNRAS, 304, 160

Kellermann, K. I., Pauliny-Toth, I. I. K., \& Williams, P. J. S. 1969, ApJ, 157, 1

Kühr, H., Witzel, A., Pauliny-Toth, I. I. K., et al. 1981, A\&AS, 45, 367

Machalski, J. 1998, A\&AS, 128, 153

Mitchell, K. J. 1983, Doctoral Dissertation, The Pennsylvania State University
Moffet, A. T., Gubbay, J., Robertson, D. S., et al. 1972, in External Galaxies and Quasi-Stellar Objects, ed. D. S. Evans, D. Wills, \& B. J. Wills (Dordrecht: D. Reidel Publishing Company), IAU Symp., 44, 228

Morganti, R., Killeen, N. E. B., \& Tadhunter, C. N. 1993, MNRAS, 263,1023

Morisawa, K., \& Takahara, F. 1987, MNRAS, 228, 745

Murphy, D. W., Browne, I. W. A., \& Perley, R. A. 1993, MNRAS, 264, 298

Owen, F. N., \& Ledlow, M. J. 1994, in The Physics of Active Galaxies, ed. G. V. Bicknell, M. A. Dopita, \& P. J. Quinn, ASP Conf. Ser., 54,319

Peacock, J. A. 1985, MNRAS, 217, 601

Peacock, J. A., \& Gull, S. F. 1981, MNRAS, 196, 611

Rengelink, R. B., Tang, Y., de Bruyn, A. G., et al. 1997, A\&AS, 124, 259

Rowan-Robinson, M. 1977, ApJ, 213, 635

Scheuer, P. A. G., \& Readhead, A. C. S. 1979, Nature, 277, 182

Scheuer, P. A. G. 1974, MNRAS, 166, 513

Snellen, I. A. G., \& Best, P. N. 2001, MNRAS, 328, 897

Urry, C. M., \& Padovani, P. 1995, PASP, 107, 803

Vermeulen, R. C., \& Cohen, M. H. 1994, ApJ, 430, 467

Vigotti, M., Grueff, G., Perley, R., et al. 1989, AJ, 98, 419

de Vries, W. H., O'Dea, C. P., \& Barthel, P. D. 2002, NAstrR, 46, 163

Wall, J. V., \& Jackson, C. A. 1997, MNRAS, 290, L17

Wall, J. V. 1980, Phil. Trans. R. Soc. London, 296, 367

Wall, J. V., Pearson, T. J., \& Longair, M. S. 1980, MNRAS, 193, 683

Willott, C. J., Rawlings, S., Blundell, K. M., et al. 2000, MNRAS, 316,449

de Young, D. S. 1972, ApJ, 177, 573 